\title{
Metodologias para seleção de fornecedores: uma revisão da literatura
}

\author{
Joana Coelho Viana ${ }^{a *}$, Luciana Hazin Alencar ${ }^{\mathrm{b}}$ \\ a*joanacviana@hotmail.com, UFPE, Brasil \\ blhazin@ufpe.br, UFPE, Brasil
}

\begin{abstract}
Resumo
A crescente participação do fornecedor na perfomance geral da contratante tem tornado as organizações mais exigentes na escolha de seus contratados, o que ocasionou a incorporação de uma diversidade de critérios de avaliação ao processo decisório. A relevância e complexidade da decisão de contratação, por sua vez, têm incentivado a busca por novas ferramentas e técnicas de seleção de fornecedores. Nesse sentido, a presente pesquisa realizou uma revisão literária dos trabalhos desenvolvidos relacionados ao tema, na qual foram analisados artigos publicados entre os anos de 1998 e 2011. A partir dessa revisão foi possivel identificar as tendências e lacunas da literatura recente, oferecendo aos interessados direcionamento para futuras aplicações e/ou aperfeiçoamentos.
\end{abstract}

Palavras-chave

Fornecedores. Seleção de fornecedores. Processo decisório.

\section{Introdução}

0 crescente impacto do desempenho do fornecedor na performance de seus contratantes tem feito as organizações repensarem seus métodos de seleção de fornecedores. A relevância da decisão tem aumentado as exigências do processo decisório que se torna cada vez mais complexo em virtude da quantidade e natureza dos critérios considerados na avaliação. Nesse contexto, intensifica-se a busca por ferramentas e técnicas capazes de balancear diversos aspectos dentro de um conjunto de alternativas.

A capacidade das organizações em atender as necessidades de seus clientes depende cada vez mais da qualidade dos produtos e serviços das empresas contratadas. Cresce, portanto, a necessidade de fornecedores bem qualificados e, sobretudo, capazes de oferecer suporte às estratégias organizacionais. As contratantes tornam-se mais seletivas e passam a exigir muito mais dos potenciais fornecedores. Tais exigências são traduzidas na forma de critérios de avaliação, adotados para a comparação entre os diferentes candidatos.

Vale ressaltar que a incorporação de uma diversidade de critérios aumenta consideravelmente a complexidade do processo de seleção. Além do grande número de fatores de avaliação, o processo lida ao mesmo tempo com critérios quantitativos e qualitativos e, muitas vezes, conflitantes. Daí a importância de métodos que permitam a articulação de diferentes aspectos na avaliação das várias alternativas.

A complexidade e relevância da decisão incentivaram o estudo de diferentes técnicas para a atividade de seleção de fornecedores (WEBER, 1996; ALMEIDA, 2001; MEADE; SARKIS, 2002; DOHEL, 2003; CHENA; LINB; HUANG, 2006; RAMANATHAN, 2007; TING; CHO, 2008; CARMO; BARROS NETO; DUTRA, 2011). Desse modo, foi desenvolvida uma variedade de ferramentas e técnicas no intuito de simplificar o processo decisório e aumentar a eficiência e eficácia dos resultados.

Esse artigo visa investigar na literatura os trabalhos desenvolvidos recentemente sobre o tema, identificando tendências, assim como lacunas existentes. Objetiva-se com isso disseminar as metodologias em maior destaque na literatura e, dessa forma, fornecer aos interessados conhecimento para a adoção e/ou aprimoramento dos métodos. 
0 presente trabalho está estruturado em 5 seções, a contar desta introdução. Na segunda seção, é apresentada a metodologia adotada para a realização da pesquisa. Em seguida, são discutidos assuntos relacionados à seleção de fornecedores e ao processo decisório, além de serem relatadas algumas classificações da literatura sobre o problema em questão. 0 quarto item descreve e discute os resultados da investigação, enquanto a quinta e última seção faz algumas considerações finais sobre o estudo.

\section{Metodologia}

Este estudo faz uma pesquisa exploratória no intuito de identificar características dos trabalhos realizados sobre a atividade de seleção de fornecedores e evidenciar os métodos mais recentemente desenvolvidos na literatura. Para Forza (2002), a finalidade dessa metodologia é construir uma ideia inicial sobre um tema, fornecendo base para estudos mais detalhados, isto é, para o aprimoramento das técnicas atualmente disponíveis.

A pesquisa foi realizada por meio de uma revisão bibliográfica, na qual foram levantados artigos publicados em periódicos de referência relacionados a temas de produção, pesquisa operacional e gestão da cadeia de suprimentos. Foram utilizadas como palavras-chave, entre outras: seleção de fornecedores, fornecedores, processo de seleção, suprimentos, cadeia de suprimentos, compras. Na concepção de Cauchick Miguel (2007), a pesquisa bibliográfica/ revisão da literatura permite identificar, conhecer e acompanhar o desenvolvimento de determinado campo de conhecimento, levantando perspectivas e sugestões para futuros trabalhos. Na presente pesquisa, a revisão literária foi inicialmente realizada para contextualizar o problema ora estudado, sendo em seguida utilizada para mapear trabalhos nesse campo de conhecimento e levantar hipóteses para futuras pesquisas.

Nesse sentido, foram investigados 56 artigos relacionados à atividade de seleção de fornecedores publicados entre os anos de 1998 e 2011. Todos os artigos analisados ofereciam uma modelagem para a resolução do problema em questão. Os trabalhos foram analisados com base em diferentes aspectos, tais como: ano de publicação, método utilizado, critérios considerados e metodologia adotada.

Os dados foram analisados para identificar características das ferramentas e técnicas mais recentemente estudadas, consolidar as questões mais atuais, levantar possíveis lacunas na área de conhecimento e, dessa forma, revelar direções para futuros desenvolvimentos.

\section{0 contexto da seleção de fornecedores}

A crescente participação do fornecedor na performance geral da organização decorre em grande parte da recente ênfase na construção de relacionamentos de parceria. Ao focarem em suas atividades fins (KRAUSE; PAGELL; CURKOVIC, 2001), repassando a terceiros todas as demais atividades não principais do negócio, as empresas se tornam cada vez mais dependentes do desempenho de seus fornecedores. Nesse contexto, é imprescindível promover a coordenação das operações entre as organizações, o que exige a construção de relacionamentos estreitos, confiáveis e duradouros.

A atividade de seleção de fornecedores surge a partir da decisão gerencial de subcontratar fornecimentos ou serviços necessários para a operação da empresa. De acordo com Perucia, Balestrin e Verschoore (2011), as organizações têm duas opções estratégicas: produção interna ou aquisição de componentes no mercado. Para os autores o elemento a ser analisado para a tomada dessa decisão é o custo de transação presente em qualquer relação econômica, tais como: custos de negociação e de formalização de contratos, de obtenção e de manutenção de clientes e os custos de acompanhamento de valores a receber. 0 custo de produção ou prestação de serviço de uma empresa especializada é em geral menor do que o da produção interna, permitindo a racionalização de custos. Entretanto, nessa conta deve-se contabilizar os custos de transação, que segundo Perucia, Balestrin e Verschoore (2011) se tornam mais ou menos elevados a depender do tipo de relacionamento estabelecido entre a empresa e seus fornecedores. Para eles, a confiança e a reciprocidade em uma relação interorganizacional são imprescindíveis para induzir a ação cooperada e reduzir o oportunismo entre os agentes econômicos, o que é considerado como um dos principais fatores de geração de custo de transação.

Assim, o processo de seleção de fornecedores ganha destaque à medida que se torna capaz de escolher os fornecedores que reúnam as características necessárias para a construção de parceria, permitindo dessa forma a redução dos custos de transação. A estratégia de cooperação possibilita a produção de bens e serviços a partir de um conjunto de empresas que, por sua vez, terão ao mesmo tempo os benefícios da hierarquia - melhor coordenação e menores custos de transação - e os benefícios da contratação - aumento de flexibilidade e ganhos de especialização na produção (PERUCIA; BALESTRIN; VERSCHOORE, 2011).

É consenso entre os especialistas que a construção de parcerias e a cooperação entre empresas proporcionam inúmeras vantagens para os membros de uma cadeia de suprimentos além de torná-la mais eficiente no 
atendimento ao cliente (CROOM et al., 2000; GUARNIERI; HATAKEYAMA, 2010; PERUCIA; BALESTRIN; VERSCHOORE, 2011; PIRES; SACOMANO NETO, 2010; WANKE; AFFONSO, 2011). No entanto, para que a estratégia de cooperação possa ser bem-sucedida, torna-se imprescindivel a formação de parcerias com empresas competentes, confiáveis e comprometidas com o resultado de toda a cadeia. Alencar, Almeida e Mota (2007) destacam que no contexto da gestão de projetos, aumenta-se ainda mais a necessidade de fornecedores qualificados, comprometidos com os objetivos do cliente e capazes de empreender os projetos solicitados. Na indústria da moda, um estudo realizado por Pereira et al. (2011), comparando as práticas chinesas e brasileiras, identifica o acesso aos fornecedores como um dos fatores que influenciam na flexibilidade, na velocidade e no custo de desenvolvimento de produtos.

Nesse novo cenário, o desempenho dos fornecedores passa a exercer papel muito mais relevante na qualidade dos produtos e serviços oferecidos pelas suas contratantes. A agilidade de um fornecedor pode limitar a habilidade de um fabricante em responder rapidamente às exigências do mercado (MARTIN, 2000). Por essa razão, as empresas têm reconhecido a necessidade de selecionar fornecedores competentes para atender devidamente as requisições de seus clientes (BURT; DOBLER; STARLING, 2003).

Essa nova visão tem tornado as organizações mais exigentes na contratação de fornecedores. No contexto atual, sente-se a necessidade de maior confiança sobre as capacidades da empresa fornecedora (McDERMOTT; HANDFIELD, 2000). Nesse sentido, as organizações têm lançado mão de diferentes critérios para fortalecer o processo de seleção (VONDEREMBSE; TRACEY, 1999).

As organizações, cada vez mais seletivas, traduzem as novas exigências em diferentes fatores de avaliação. Atualmente, além do preço, qualidade e entrega, diversos outros critérios, tangíveis e intangíveis, vêm sendo considerados no processo de seleção de fornecedores, tais como: capacidade tecnológica, compromisso, credibilidade, cultura organizacional, histórico de performances, dentre outros. Neumann e Ribeiro (2004) ressaltam que algumas vezes é necessário um confirmação local de certas características, uma vez que a percepção da empresa contratante pode não refletir a real situação do fornecedor.

A diversidade de critérios de avaliação tem aumentado a complexidade do processo seletivo. 0 problema da escolha do fornecedor torna-se mais complicado quando se consideram aspectos relacionados às particularidades de produtos e/ou situações (VOKURKA; CHOOBINEH; VADI, 1996). Entretanto, Kakouris, Polychronopoulos e Binioris (2006) ressaltam que o conjunto adequado de critério deve refletir as peculiaridades de cada operação.

\subsection{Métodos de seleção de fornecedores}

A quantidade e natureza dos critérios avaliados complicam o processo seletivo. A adoção de fatores qualitativos dificulta a sua mensuração devido ao caráter eminentemente subjetivo. Além disso, é comum a existência de aspectos conflitantes, como qualidade e preço que precisam ser balanceados.

A complexidade do processo seletivo requer, portanto, ferramentas e técnicas capazes tanto de facilitar o processo decisório como de aumentar a eficiência da escolha. Uma das partes mais difíceis desse processo decisório é a definição dos parâmetros, uma vez que estes irão refletir as preferências e os trade-offs dos decisores (ALENCAR; ALMEIDA, 2008). Após essa definição, a articulação de todos os critérios considerados se torna uma das grandes preocupações dos estudiosos, que vêm trabalhando no desenvolvimento de metodologias específicas para a resolução do problema de seleção.

$\mathrm{Na}$ literatura, diversas modelagens foram desenvolvidas para a atividade de seleção de fornecedores, as quais utilizam desde modelos de ponderação até métodos mais sofisticados com programação matemática e métodos de apoio a decisão multicritério. Os modelos propostos visam englobar o máximo de critérios possíveis e reduzir a subjetividade da decisão. Alguns desses modelos abrangem a decisão em grupo, existindo um grande número de ferramentas para tratar esse tipo de problema (DAHER; ALMEIDA, 2010).

$A$ variedade das ferramentas e técnicas para a solução do problema em questão é bastante extensa. Nesse item, entretanto, serão brevemente descritos apenas os métodos discutidos no decorrer do presente trabalho. São eles:

- Ponderação linear: nesse modelo, pesos são atribuídos a cada critério, geralmente de forma subjetiva. A avaliação global de cada fornecedor é dada pela soma do seu desempenho nos critérios multiplicado pelos seus respectivos pesos (WEBER; CURRENT; BENTON, 1991);

- Programação matemática: o problema é formulado em uma função matemática objetiva a qual posteriormente é maximizada ou minimizada (DE BOER; LABRO; MORLACCHI, 2001);

- Modelo multicritério: ajuda o decisor a sistematicamente avaliar um conjunto de alternativas em relação a vários critérios (VINCKE, 1992);

- Total cost of ownership (TCO): incorpora, na escolha do fornecedor, todos os custos mensuráveis incorridos durante o ciclo de vida do item comprado (DEGRAEVE; ROODHOOFT, 1999);

- Data envelopment analysis (DEA): as alternativas são avaliadas de acordo com os critérios de benefício 
(outputs) e os critérios de custo (inputs). A eficiência de uma alternativa, no caso de um fornecedor, é obtida através da razão da soma ponderada de seus outputs pela soma ponderada de seus inputs (DE BOER; LABRO; MORLACCHI, 2001);

- Teoria fuzzy: valores linguísticos são expressos em números fuzzy, sendo utilizados para avaliar e atribuir pesos aos critérios (CHENA; LINB; HUANG, 2006); e

- Inteligência artificial: são baseados em sistemas computacionais (DE BOER; LABRO; MORLACCHI, 2001).

\subsection{Outras classificações da literatura de seleção de fornecedores}

Diante da diversidade de modelos disponiveis para o problema de seleção de fornecedores, alguns estudos surgiram no intuito de levantar e analisar a literatura existente sobre o assunto. 0 objetivo desses trabalhos é contribuir com o desenvolvimento da área, fornecendo uma visão das ferramentas e técnicas propostas bem como direcionamento para futuros estudos e aperfeiçoamentos.

Já em 1991, Weber, Current e Benton investigou 74 artigos sobre o assunto publicados desde 1966, classificando-os de acordo com o método proposto, os critérios considerados e o ambiente de compras em que a empresa estava inserida. 0 foco do estudo se concentrou na classificação dos artigos com base na relação de 23 critérios levantados em um estudo anteriormente realizado por Dickson em 1966. Os trabalhos também foram categorizados de acordo com a técnica ou método analítico utilizado para avaliar os critérios. Nesta época, a maioria dos modelos propostos se concentrava na aplicação da abordagem de ponderação linear.

De Boer, Labro e Morlacchi (2001), por sua vez, fizeram uma revisão sobre métodos de suporte para a seleção de fornecedores, considerando as diversas etapas do processo, desde a formulação do problema, passando pela formulação dos critérios, qualificação, até a seleção final. Concluíram que fatores como o número de fornecedores disponíveis, a importância da compra e/ou do relacionamento com o fornecedor e a quantidade de incerteza presente determinam o método mais adequado a ser utilizado em cada situação, enfatizando a influência da característica da compra (nova compra, recompra modificada, recompra de rotina ou recompra estratégica) sobre esses aspectos. Os autores classificaram cada trabalho em relação à fase do processo de seleção de fornecedores correspondente ao modelo proposto, levando em consideração a característica da compra.
Em 2002, Bhutta e Huq realizou um trabalho extenso acerca da literatura existente sobre a seleção de fornecedores. Em seu estudo foram revisados 154 artigos de 68 periódicos entre os anos de 1986 a 2002. Os artigos analisados foram classificados de acordo com a abordagem e a metodologia utilizada. Além disso, o autor identificou os periódicos que apresentam publicações sobre o assunto e levantou a frequência anual de publicação da literatura de seleção de fornecedores em cada um. 0 trabalho de Bhutta e Huq (2002) proporcionou uma visão sobre os métodos em maior destaque na literatura na época, oferecendo insights para futuros trabalhos.

No intuito de dar continuidade a esses trabalhos, o presente artigo faz uma nova revisão, considerando publicações de 1998 até 2011. 0 objetivo é continuar fornecendo um direcionamento aos estudos de pesquisadores e profissionais interessados no assunto.

\section{Resultados e discussões}

Os 56 artigos investigados foram analisados e classificados a partir de diferentes aspectos. Os resultados encontrados e as discussões sobre cada um dos aspectos estudados são apresentados a seguir.

\subsection{Frequência dos artigos}

Para a realização da presente pesquisa, foram selecionados trabalhos publicados entre os anos de 1998 e 2011. A Figura 1 expõe a distribuição das publicações no decorrer do período investigado. De acordo com os dados, observa-se que grande parte dos artigos em análise foram publicados nos anos de 2007, 2008 e 2011, período no qual se concentram quase $48 \%$ das publicações.

Apesar do número reduzido de publicações relacionadas ao tema nos anos de 2009 e 2010, observa-se que quase 65\% dos artigos foram publicados a partir de 2006. Isto posto, a concentração de publicações nos últimos anos sugere a existência de um novo direcionamento ao problema de seleção de fornecedores e destaca a relevância dessa atividade no

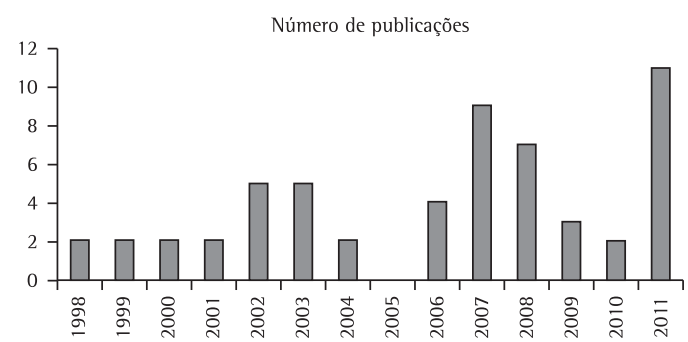

Figura 1. Número de publicações por ano. Fonte: o autor (2011). 
contexto atual. Esse resultado permite a construção da hipótese a seguir:

- $\mathrm{H}_{1}=$ Há um novo direcionamento de trabalhos acerca do processo de seleção de fornecedores.

\subsection{Critérios de avaliação}

As organizações, cada vez mais seletivas, têm adotado uma série de critérios na escolha de seus fornecedores. Em decorrência dessa prática, os modelos de seleção propostos na literatura englobam diferentes atributos que variam de fatores quantitativos - como preço e quantidade de entregas no prazo - até fatores qualitativos, que são bem mais difíceis de mensurar - como o compromisso do fornecedor e sua habilidade de solucionar problemas.

Todos os trabalhos analisados contemplaram em sua metodologia pelo menos 2 aspectos de avaliação. Destes, 13\% incluíram até 3 atributos no modelo proposto, enquanto mais da metade, $66 \%$, considerou mais de 5 fatores. Verifica-se um elevado percentual de artigos, 18\%, cuja modelagem desenvolvida abrange mais de 10 critérios distintos. Vale destacar que houve modelo que sugeriu a utilização de mais de 20 fatores de avaliação. A Tabela 1 mostra a distribuição das publicações de acordo com o número de critérios empregados. Com base nos dados obtidos, constitui-se a seguinte proposição:

- $\mathrm{H}_{2}=0$ s modelos recentes de seleção de fornecedores englobam pelo menos 5 critérios de avaliação.

A partir da análise dos artigos, foi constituída uma relação de 70 fatores de avaliação. 0 Quadro 1 relaciona os aspectos mais considerados nas metodologias pesquisadas. Observa-se que os atributos preço, qualidade e entrega são considerados na grande maioria dos modelos propostos, se mantendo em destaque em relação aos demais critérios. É possível perceber a incorporação de fatores qualitivos, tais como: capacidade tecnológica $(37,50 \%)$, capacidade de relacionamento/cooperação $(16,07 \%)$, flexibilidade $(17,86 \%)$, credibilidade $(12,50 \%)$

Tabela 1. Número de critérios considerados.

\begin{tabular}{cc}
\hline Número de critérios & $\%$ \\
\hline 2 & 2 \\
3 & 12 \\
4 & 19 \\
5 & 20 \\
6 & 11 \\
7 & 11 \\
8 & 2 \\
9 & 2 \\
$10 \mathrm{ou}+$ & 18 \\
\hline
\end{tabular}

Fonte: o autor (2011). e gestão e organização (12,50\%). Verifica-se ainda a aplicação de boa parte dos critérios listados na relação de 23 atributos de Dickson, configurada em 1966. Dos 14 critérios mais citados, apenas 4 não fazem parte dessa relação: serviço ao cliente, capacidade de relacionamento/cooperação, práticas e sistemas de gestão de qualidade e diversidade de itens.

Apesar da grande atenção dada a fatores relacionados com o meio ambiente, apenas 3 trabalhos analisados consideraravam aspectos de responsabilidade social e ambiental (BÜYÜKÖZKAN; ÇIFÇıl, 2011; HANDFIELD et al., 2002; YEH; CHUANG, 2011).

Os resultados encontrados com essa análise levantaram a hipótese a seguir:

- $\mathrm{H}_{3}=\mathrm{Na}$ seleção de fornecedores, são considerados outros critérios além do preço, qualidade e entrega.

Esses resultados evidenciam a incorporação de diferentes aspectos de análise no processo seletivo, cuja aplicação vem sendo possibilitada pelo desenvolvimento de métodos que lidem com múltiplos critérios.

\subsection{Metodologias de estudo}

Os trabalhos diferem entre si também quanto à metodologia empregada para a elaboração do estudo. Nesse sentido, a literatura revisada foi classificada em duas categorias distintas, adaptadas de um trabalho anteriormente realizado por Buttha e Huq (2002):

- Estudos metodológicos: fornecem métodos para solucionar o problema de seleção de fornecedores, mas não apresentam sua aplicação ou a apresentam utilizando exemplos hipotéticos;

- Estudos de caso: fornecem detalhes da aplicação do modelo proposto em exemplos reais de empresas.

Nesse item, a categorização dos trabalhos foi de certa forma equilibrada. Foram classificados como estudos metodológicos aproximadamente $60 \%$ das publicações analisadas, ao passo que $40 \%$ foram qualificadas como estudos de caso.

Os trabalhos de estudo de caso demonstram os modelos propostos em diversos contextos. Os exemplos ilustrados são aplicados a diferentes setores da economia, tais como: telecomunicações, farmacêutico, químico, de vestuário, automobilística, eletrônicos, entre vários outras. Este fato evidencia o crescente interesse dos estudiosos em demonstrar os aspectos práticos de seus modelos e disseminar as ferramentas e técnicas desenvolvidas. Com esses dados é possível construir a suposição a seguir:

- $\mathrm{H}_{4}=$ Os estudiosos estão se preocupando com a aplicação dos modelos desenvolvidos em situações reais. 


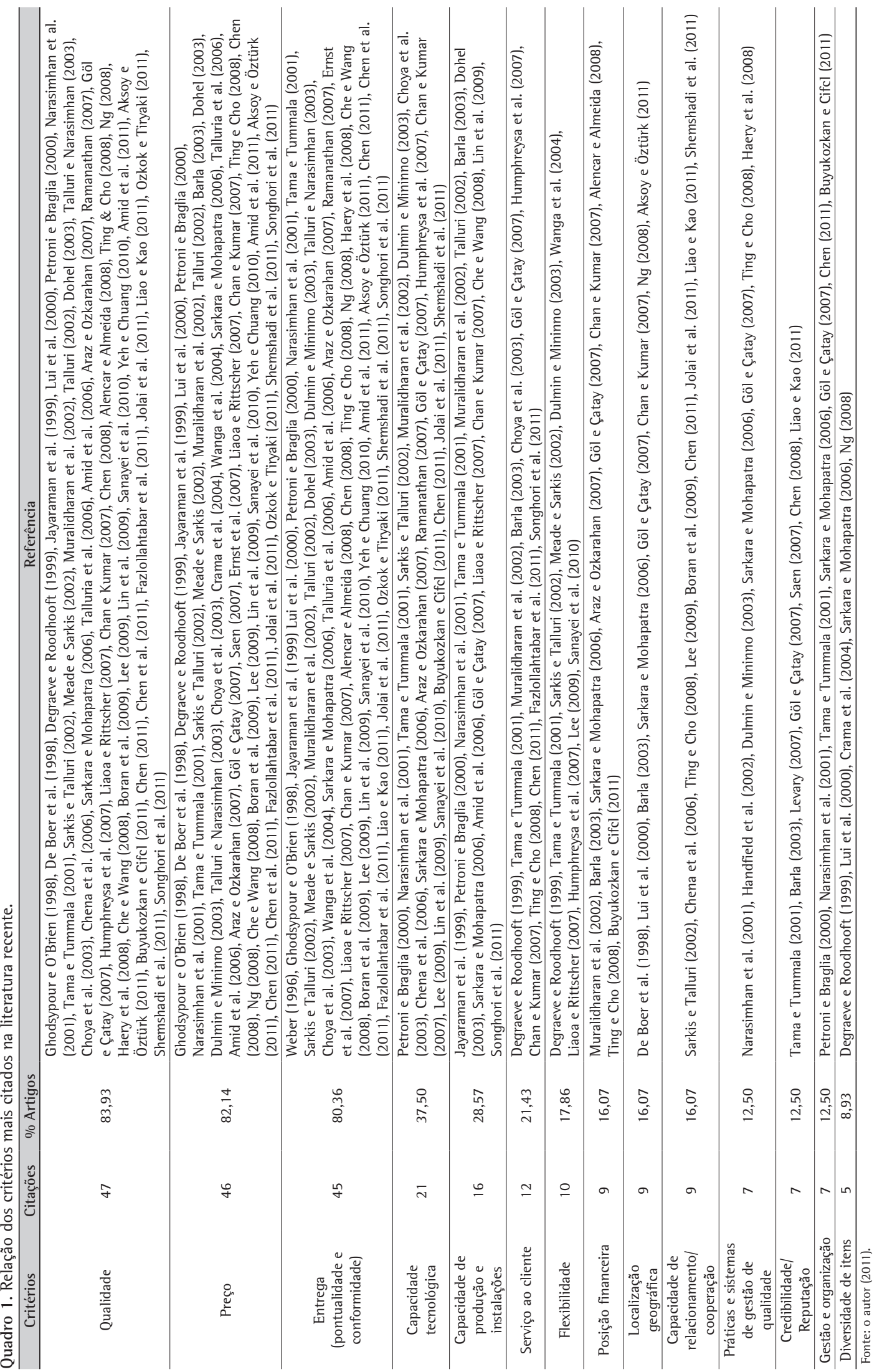




\subsection{Métodos propostos}

Ao longo dos anos, foram desenvolvidos diversos modelos de seleção de fornecedores com o intuito de proporcionar soluções mais eficientes à problemática. Tais modelos foram sendo adaptados às novas circunstâncias e exigências do mercado, o que proporcionou o aprimoramento de técnicas já disponíveis, assim como a criação de novas ferramentas.

A necessidade de avaliar múltiplos critérios transformou o problema em uma decisão multicritério e aumentou a complexidade do processo decisório. Esse fato estimulou a busca por métodos de fácil entendimento e estruturação, que ao mesmo tempo fossem eficientes na consolidação dos critérios e na comparação das alternativas.

Atualmente, a diversidade de modelagens, para a estruturação e resolução do problema, abrange desde simples categorização até métodos mais sofisticados de apoio à decisão multicritério. 0 Quadro 2 relaciona as metodologias típicas adotadas na construção de modelos para a seleção de fornecedores.

É importante destacar que, devido à complexidade do processo decisório, alguns trabalhos têm construído seus modelos com base em uma combinação de duas ou três técnicas. Dentre as publicações investigadas, aproximadamente $43 \%$ apresentaram uma modelagem constituída por mais de um método, o que representa um percentual significativo.

A Figura 2 ilustra o percentual dos trabalhos que adotaram cada uma das metodologias relacionadas anteriormente. Evidencia-se a predominância dos métodos de apoio à decisão multicritério, que aparecem em 50\% das publicações analisadas. Em seguida, destacam-se os modelos com base na teoria fuzzy $(25 \%)$, que aparecem predominantemente nos trabalhos mais recentes. Cerca de 79\% dos métodos que envolvem a teoria fuzzy foram propostos nos últimos três anos. A programação matemática compõe $23 \%$ das propostas de modelagem, as quais incluem programação linear, linear multiobjetivo, inteira mista, inteira mista multiobjetivo ou dinâmica. A técnica data envelopment analysis (DEA) é aplicada em 12,5\% dos artigos investigados, enquanto 7\% dos trabalhos utilizaram métodos classificados como inteligência artificial. As demais abordagens foram empregadas em menos de 7\% das modelagens. Esses resultados remetem às seguintes proposições:

- $\mathrm{H}_{5}=$ As novas modelagens de seleção de fornecedores tendem a incorporar mais de um método de decisão.

- $\mathrm{H}_{6}=0$ s modelos de seleção de fornecedores envolvem métodos de apoio à decisão multicritério.

Considerando as abordagens de apoio à decisão multicritério, a Figura 3 expõe os métodos mais adotados. Verifica-se que, dentre os 50\% das propostas de modelo multicritérios, quase metade (43\%) sugere a aplicação do método AHP (Analytic Hierarchy Process), percentual significativo também em relação a todas as modelagens analisadas, compreendendo $21,4 \%$ do total. 0 ANP (Analytic Network Process) foi adotado em 4 dos modelos investigados. A Família ELECTRE (Elimination and Choice Translating Algorithm) e a Família PROMETHEE (Preference Ranking Method for Enrichment Evaluation) foram empregadas em apenas 2 trabalhos, cada.

\subsection{Discussões}

Com base na investigação realizada neste estudo, percebe-se uma renovação do interesse de estudiosos na atividade de seleção de fornecedores. Foi observado

Quadro 2. Resumo de metodologias para a seleção de fornecedores.

\begin{tabular}{|c|c|}
\hline Método & Referência \\
\hline $\begin{array}{l}\text { Método de apoio à decisão } \\
\text { multicritério }\end{array}$ & $\begin{array}{l}\text { Ghodsypour e O’Brien (1998), De Boer et al. (1998), Tama e Tummala (2001), Meade e Sarkis (2002), } \\
\text { Sarkis e Talluri (2002), Muralidharan et al. (2002), Handfield et al. (2002), Barla (2003), Dulmin e Mininno } \\
\text { (2003), Wanga et al. (2004), Chena et al. (2006), Araz e Ozkarahan (2007), Levary (2007), Ramanathan } \\
\text { (2007), Göl e Çatay (2007), Chan e Kumar (2007), Alencar e Almeida (2008), Ting e Cho (2008), } \\
\text { Boran et al. (2009), Lee (2009), Lin et al. (2009), Sanayei et al. (2010), Amid et al. (2011), Chen (2011), } \\
\text { Buyukozkan e Cifcl (2011), Fazlollahtabar et al. (2011), Liao e Kao (2011), Shemshadi et al. (2011) }\end{array}$ \\
\hline Teoria fuzzy & $\begin{array}{l}\text { Sarkara e Mohapatra (2006), Amid et al. (2006), Chena et al. (2006), Chan e Kumar (2007), Boran et al. } \\
\text { (2009), Lee (2009), Sanayei et al. (2010), Amid et al. (2011), Buyukozkan e Cifcl (2011), Chen (2011), } \\
\text { Chen et al. (2011), Jolai et al. (2011), Liao e Kao (2011), Ozkok e Tiryaki (2011), Shemshadi et al. (2011) }\end{array}$ \\
\hline Programação matemática & $\begin{array}{l}\text { Ghodsypour e O'Brien (1998), Degraeve e Roodhooft (1999), Jayaraman et al. (1999), Dohel (2003), } \\
\text { Wanga et al. (2004), Crama et al. (2004), Liaoa e Rittscher (2007), Ting e Cho (2008), Chen et al. (2011), } \\
\text { Fazlollahtabar et al. (2011), Jolai et al. (2011), Liao e Kao (2011), Ozkok e Tiryaki (2011), Songhori et al. (2011) }\end{array}$ \\
\hline Data envelopment analysis (DEA) & $\begin{array}{l}\text { Weber (1996), Liu et al. (2000), Narasimhan et al. (2001), Talluria et al. (2006), Saen (2007), Ramanathan } \\
\text { (2007), Chen (2011), Songhori et al. (2011) }\end{array}$ \\
\hline Métodos estatísticos & Petroni e Braglia (2000), Talluri e Narasimhan (2003), Ernst (2007) \\
\hline Ponderação linear & Humphreysa et al. (2007), Chen (2008), Ng (2008) \\
\hline Inteligência artificial & Choya et al. (2003), Che e Wang (2008),Yeh e Chuang (2010) Aksoy e Öztürk (2011) \\
\hline Total cost of ownership (TCO) & Degraeve e Roodhooft (1999), Ramanathan (2007) \\
\hline Data mining & Haery et al. (2008) \\
\hline
\end{tabular}

Fonte: o autor (2011). 


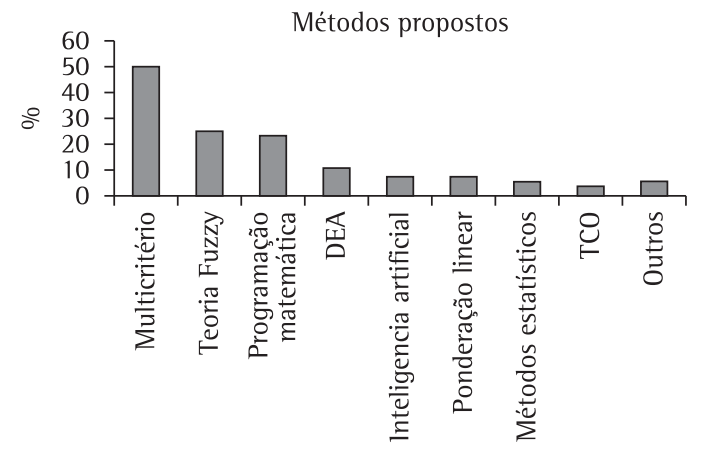

Figura 2. Métodos propostos. Fonte: o autor (2011).

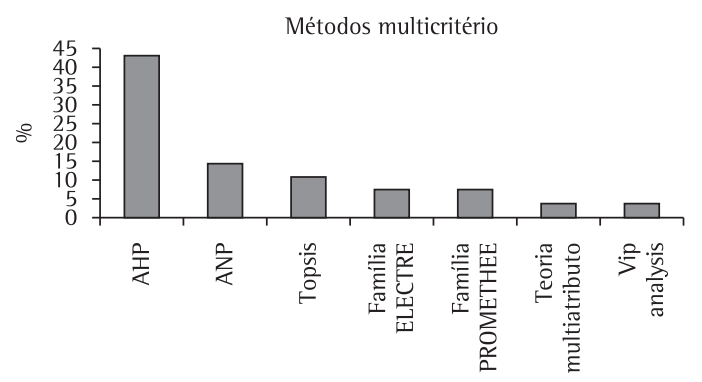

Figura 3. Métodos multicritério. Fonte: o autor (2011).

um crescimento no número de publicações ao longo dos anos, com destaque para os cinco últimos anos, período no qual foram publicados quase $65 \%$ dos trabalhos investigados. Pressupõe-se que o ressurgimento desse interesse tenha sido motivado pela complexidade do processo decisório aliada à importância da decisão no atual ambiente de negócios.

0 volume de trabalhos relacionados ao tema encontrados na literatura reforça a relevância do problema de seleção. A evolução dos conceitos da Gestão da Cadeia de Suprimentos defende o estabelecimento de relações de parceria entre empresas compradoras e fornecedoras, aumentando as responsabilidades dessas últimas sobre os resultados das primeiras. Essa visão tem sido incorporada aos novos modelos de gestão que têm reconhecido cada vez mais o impacto do desempenho do fornecedor nas operações organizacionais. Verma e Pullman (1998) já destacavam a crescente relevância das questões de seleção de fornecedores em virtude do aumento da implementação dos conceitos da Gestão Qualidade Total (TQM) e do Just-in-time (JIT) em um grande número de empresas.

As consequências da crescente participação dos fornecedores na performance geral de suas contratadas são o aumento do nível de exigência adotados na seleção e a necessidade de processos mais bem estruturados.

As empresas, mais seletivas em suas contratações, passaram a avaliar uma quantidade maior de aspectos em cada potencial fornecedor. 0 número de critérios adotados nas modelagens investigadas reflete essa tendência. Mais da metade dos modelos propostos, $66 \%$, empregam no mínimo 5 critérios ao passo que uma parcela considerável de propostas, 18\%, lidam com 10 ou mais aspectos diferentes.

A mudança de comportamento das empresas em relação a seus fornecedores também repercutiu no tipo de fatores de avaliação aplicados no processo decisório. Por meio desta pesquisa, foi possível observar a variedade de critérios, quantitativos e qualitativos, empregados atualmente. A revisão dos artigos resultou em uma relação inicial de 70 atributos. Supõe-se que os modelos desenvolvidos estão adotando critérios mais específicos, levando em consideração as circunstâncias do problema estudado, conforme sugerido por Vokurka, Choobineh e Vadi (1996) e Kakouris, Polychronopoulos e Binioris (2006).

Nesta investigação, confirma-se a incorporação de novos critérios além dos tradicionais: preço, qualidade e entrega. Entre os mais citados estão: capacidade de produção, capacidade tecnológica, serviços oferecidos, flexibilidade, posição financeira, dentre outros. É importante destacar a presença de critérios como credibilidade/reputação e capacidade de relacionamento/cooperação, que evidenciam a recente preocupação das organizações com a confiança e o compromisso do fornecedor, seguindo a tendência da construção de parcerias na cadeia de suprimentos. Verifica-se que parte dos fatores de avaliação mais utilizados nos modelos analisados coincidem com os mesmos encontrados no estudo de Weber, Current e Benton (1991). Isso reforça o fato de que as novas metodologias de seleção de fornecedores não substituíram os critérios inicialmente aplicados, mas acrescentaram atributos decorrentes das novas exigências do mercado e peculiaridades do negócio.

Ao passo que as requisições das contratantes são traduzidas em fatores de avaliação, surge a necessidade de processos seletivos estruturados, capazes de lidar com múltiplos critérios e, sobretudo, eficientes na escolha. A busca por novas ferramentas e técnicas incentivou o estudo e o desenvolvimento de métodos, resultando no surgimento de uma ampla diversidade de modelos, que muitas vezes utilizam mais de uma técnica. Os trabalhos analisados nesta pesquisa lançaram mão de métodos de apoio à decisão multicritério, teoria fuzzy, programação matemática, data envelopment analysis (DEA), métodos estatísticos, inteligência artificial, dentre outros. Foi observada uma predominância dos métodos de apoio à decisão multicritério, os quais foram utilizados em 50\% dos trabalhos. Esse resultado mostra uma mudança de tendência em relação ao que se vinha trabalhando na literatura entre os anos de 1986 e 2002, cujas publicações foram analisadas no estudo de Bhutta e Huq (2002). Nesse estudo, a técnica de total cost of ownership (TCO) teve o maior destaque, compreendendo 33\% dos trabalhos investigados, seguida de outras abordagens de custo total, que 
foram empregadas em 19\% dos artigos. Nessa época, os métodos de apoio à decisão multicritério foram adotados em apenas 22\% das publicações. Assim, sugere-se que a recente necessidade de contrabalancear múltiplos critérios, às vezes contraditórios, tenha provocado esse direcionamento.

Embora, recentemente, a metodologia multicritério tenha sido bastante utilizada no desenvolvimento de modelos, pouco foi explorado dos seus métodos. De acordo com Szajubok, Alencar e Almeida (2006), essa área do conhecimento possui um amplo conjunto de ferramentas, cujo objetivo é dar suporte no desenvolvimento de um processo de decisão.

Dentre as modelagens propostas com base nessa metodologia, quase metade (43\%) empregou o AHP. 0 método ANP, que é um avanço do AHP, foi adotado em 4 dos modelos analisados. Apenas 2 trabalhos aplicaram métodos da família PROMETHEE, e outros dois, da família ELECTRE. A teoria multiatributo e o VIP analysis foram adotados em um único trabalho, cada. Esse resultado evidencia um espaço para o desenvolvimento de modelos que contemplem técnicas mais avançadas de apoio à decisão multicritério.

No que diz respeito aos passos do processo seletivo, De Boer, Labro e Morlacchi (2001) haviam indicado o direcionamento dos trabalhos para a etapa de seleção (escolha) em detrimento das fases iniciais do processo, tais como identificação dos critérios e respectivos pesos. Contudo, nessa pesquisa, foi visto que grande parte dos modelos propostos já compreende ambas as etapas. Ressalta-se, entretanto, que poucos trabalhos focaram a forma de mensuração dos fatores de avaliação que representa uma etapa delicada do processo. Para a atribuição de pesos e medição dos critérios de julgamento, boa parte dos trabalhos (25\%), inclusive os mais recentes, tem lançado mão de abordagens com base na teoria fuzzy.

\section{Considerações finais}

A presente pesquisa investigou, na literatura recente, publicações relacionadas à problemática de seleção de fornecedores. Nessa revisão, foram identificadas as tendências e as lacunas de trabalhos desenvolvidos nesse campo de conhecimento, conforme previsto no objetivo deste estudo. Foram evidenciadas as técnicas mais adotadas por especialistas na construção de modelagens para o processo de seleção de fornecedores, assim como foram destacadas ferramentas ainda pouco exploradas pelos estudiosos, mas que parecem oferecer um potencial de soluções para o problema. Dessa forma, os resultados obtidos fornecem aos interessados direcionamento para a aplicação e/ou aprimoramento de modelos de seleção.

Os resultados encontrados mostram uma renovação do interesse de estudiosos acerca do processo de seleção de fornecedores, em que se observa um crescimento, ao longo dos anos, no número de publicações com propostas para a resolução do problema, destacando a relevância do assunto. As modelagens desenvolvidas, por sua vez, revelam a adequação dos modelos às novas exigências, sendo incorporados ao processo decisório mais fatores além do preço, qualidade e entrega, incluindo-se fatores qualitativos e, muitas vezes, conflitantes. Esse aspecto reflete na construção dos modelos, nos quais são empregados, especialmente, os métodos de apoio à decisão multicritério. No entanto, vale destacar que pouco tem sido explorado das ferramentas de apoio à decisão multicritério. Métodos como os das famílias PROMETHEE e ELECTRE, teoria multiatributo, dentre outros, foram pouco utilizados.

É importante destacar que a investigação realizada analisou características gerais dos artigos, não compreendendo uma análise detalhada dos modelos propostos em relação à estruturação, eficiência, situações indicadas e objetivos prioritários. Além disso, não foram consideradas as circunstâncias do ambiente no qual cada modelo foi aplicado, fator relevante na elaboração das metodologias.

Sugere-se, portanto, para futuros trabalhos o teste das hipóteses levantadas na presente pesquisa. Além disso, pode ser realizada uma avaliação mais aprofundada das modelagens propostas, na qual fosse possivel identificar características, peculiaridades e condições para a aplicação de cada uma delas. As particularidades do ambiente de mercado também podem ser avaliadas, como a disponibilidade de fornecedores, característica da compra, concorrência, velocidade de mudanças, dentre outros aspectos. Essa visão proporciona ao decisor mecanismos para averiguar a adequação de um modelo em cada contexto, podendo também contribuir para o aprimoramento das ferramentas e técnicas disponíveis.

\section{Referências}

ALENCAR, L. H.; ALMEIDA, A. T. Multicriteria decision group model for the selection of suppliers. Pesquisa Operacional, v. 28, n. 2, p. 321-337, 2008. http://dx.doi.org/10.1590/ S0101-74382008000200009

ALENCAR, L. H.; ALMEIDA, A. T.; MOTA, C. M. M. Sistemática proposta para seleção de fornecedores em gestão de projetos. Gestão e Produção, v. 14, n. 3, p. 477-487, 2007.

ALMEIDA, A. T. Multicriteria Decision Making on Maintenance: Spares and Contracts Planning. European Journal of Operational Research, v. 129, n. 2, p. 235-241, 2001. http://dx.doi.org/10.1016/S0377-2217(00)00220-4

AMID, A.; GHODSYPOURA, S. H.; O'BRIEN, C. Fuzzy multiobjective linear model for supplier selection in a supply chain. International Journal of Production Economics, v. 104, p. 394-407, 2006. http://dx.doi. $\operatorname{org} / 10.1016 /$ j.ijpe.2005.04.012

AMID, A.; GHODSYPOURA, S. H.; O’BRIEN, C. A weighted maxmin model for fuzzy multi-objective supplier selection in a supply chain. International Journal Production Economics, v. 131, p. 139-145, 2011. http://dx.doi.org/10.1016/j. ijpe.2010.04.044 
AKSOY, A.; ÖZTÜRK, N. Supplier selection and performance evaluation in just-in-time production environments. Expert Systems with Applications, v. 38, p. 63516359, 2011. http://dx.doi.org/10.1016/j.eswa.2010.11.104

ARAZ, C.; OZKARAHAN, 1. Supplier evaluation and management system for strategic sourcing based on a new multicriteria sorting procedure. International Journal of Production Economics, v. 106, p. 585-606, 2007. http://dx.doi. org/10.1016/j.ijpe.2006.08.008

BARLA, S. B. A case study of supplier selection for lean supply by using a mathematical model. Logistics Information Management, v. 16, n. 6, p. 451-459, 2003. http://dx.doi. org/10.1108/09576050310503420

BORAN, F. E. et al. A multi-criteria intuitionistic fuzzy group decision making for supplier selection with TOPSIS method. Expert Systems with Applications, v. 36, p. 11363-11368, 2009. http://dx.doi.org/10.1016/j. eswa.2009.03.039

BURT, D. N.; DOBLER, D. W.; STARLING, S. L. World Class Supply Management: The key to Supply Chain Management. 7th ed. New York: McGraw-Hill, 2003.

BUTTHA, K. S.; HUQ, F. Supplier selection problem: a comparison of total cost of ownership and analytic hierarchy process approaches. Supply Chain Management: An International Journal, v. 7, n. 3, p. 126-135, 2002. http://dx.doi.org/10.1108/13598540210436586

BÜYÜKÖZKAN, G.; ÇIFÇl, G. A novel fuzzy multi-criteria decision framework for sustainable supplier selection with incomplete information. Computers in Industry, v. 62, p. 164-174, 2011. http://dx.doi.org/10.1016/j. compind.2010.10.009

CARMO, B. B. T.; BARROS NETO, J. F.; DUTRA, N. G. S. Análise do impacto nos custos de transporte de um modelo de seleção de fornecedores baseado em variáveis socioambientais e de competitividade. Produção, v. 21, n. 3, p. 466-483, 2011. http://dx.doi.org/10.1590/S010365132011005000013

CAUCHICK MIGUEL, P. A. Estudo de caso na engenharia de produção: estruturação e recomendações para sua condução. Produção, v. 17, n. 1, p. 216-229, 2007.

CHAN, F. T. S.; KUMAR, N. Global supplier development considering risk factors using fuzzy extended AHP-based approach. Omega, v. 35, p. 417-431, 2007. http://dx.doi. org/10.1016/j.omega.2005.08.004

CHE, Z. H.; WANG, H. S. Supplier selection and supply quantity allocation of common and non-common parts with multiple criteria under multiple products. Computers \& Industrial Engineering, v. 55, p. 110-133, 2008. http:// dx.doi.org/10.1016/j.cie.2007.12.005

CHEN, C. C. A model for customer-focused objectivebased performance evaluation of logistics service providers. Asia Pacific Journal of Marketing and Logistics, v. 20, n. 3, p. 309-322, 2008. http://dx.doi. org/10.1108/13555850810890075

CHEN, T. Y.; WANG, H. P.; LU, Y. Y. A multicriteria group decision-making approach based on interval-valued intuitionistic fuzzy sets: A comparative perspective. Expert Systems with Applications, v. 38, p. 7647-7658, 2011. http://dx.doi.org/10.1016/j.eswa.2010.12.096

CHEN, Y. J. Structured methodology for supplier selection and evaluation in a supply chain. Information Sciences, v. 181, p. 1651-1670, 2011. http://dx.doi.org/10.1016/j. ins.2010.07.026

CHENA, C. T.; LINB, C. T.; HUANG, S. F. A fuzzy approach for supplier evaluation and selection in supply chain management. International Journal of Production Economics, v. 102, p. 289-301, 2006. http://dx.doi. org/10.1016/j.ijpe.2005.03.009
CHOYA, K. L.; LEEA, W. B.; LO, V. Design of a case based intelligent supplier relationship management system - the integration of supplier rating system and product coding system. Expert Systems with Applications, v. 25, p. 87100, 2003.

CRAMA, Y.; PASCUAL, J. P.; TORRES, A. Optimal procurement decisions in the presence of total quantity discounts and alternative product recipes. European Journal of Operational Research, v. 159, p. 364-378, 2004. http:// dx.doi.org/10.1016/j.ejor.2003.08.021

CROOM, S. et al. Supply chain management: an analytical framework for critical literature review. European Journal of Purchasing \& Supply Management, v. 6, p. 67-83, 2000. http://dx.doi.org/10.1016/S0969-7012(99)00030-1

DAHER, S. F. D.; ALMEIDA, A. T. Recent patents using group decision support systems: A Short Review. Recent Patents on Computer Science, v. 3, p. 81-90, 2010.

DE BOER, L.; LABRO, E.; MORLACCHI, P. A review of methods supporting supplier selection. European Journal of Purchasing \& Supply Management, v. 7, n. 2, p. 75-89, 2001. http://dx.doi.org/10.1016/S09697012(00)00028-9

DE BOER, L.; WEGEN, L. V.; TELGEN, J. Outranking methods in support of supplier selection. European Journal of Purchasing \& Supply Management, v. 4, p. 109-118, 1998. http://dx.doi.org/10.1016/S0969-7012(97)00034-8

DEGRAEVE, Z.; ROODHOOFT, F. Effectively selecting suppliers using total cost of ownership. Journal of Supply Chain Management, v. 35, n. 1, p. 5-10, 1999. http://dx.doi. org/10.1111/j.1745-493X.1999.tb00050.x

DICKSON, G.W. An analysis of vendor selection systems and decision. Journal of Purchasing, v. 2, n. 1, p.5-17, 1966.

DOHEL, N. Vendor selection and order quantity allocation in volume discount environments. Supply Chain Management: An International Journal, v. 8, n. 4, p. 335-342, 2003. http://dx.doi.org/10.1108/13598540310490099

DULMIN, R.; MININNO, V. Supplier selection using a multicriteria decision aid method. Journal of Purchasing \& Supply Management, v. 9, p. 177-187, 2003 http://dx.doi. org/10.1016/S1478-4092(03)00032-3

ERNST, R.; KAMRAD, B.; ORD, K. Delivery performance in vendor selection decisions. European Journal of Operational Research, v. 176, p. 534-541, 2007 http:// dx.doi.org/10.1016/j.ejor.2005.03.051

FORZA, C. Survey research in operations management: a processbased perspective. International Journal of Operations \& Production Management, v. 22, n. 2, p. 152-194, 2002. http://dx.doi.org/10.1108/01443570210414310

FAZLOLLAHTABAR, $\mathrm{H}$. et al. A multi-objective decision-making process of supplier selection and order allocation for multiperiod scheduling in an electronic market. International Journal Advanced Manufacturing Technology, v. 52, p. 1039-1052, 2011. http://dx.doi.org/10.1007/s00170010-2800-6

GHODSYPOUR, S. H.; O'BRIEN, C. A decision support system for supplier selection using an integrated analytic hierarchy process and linear programming. International Journal of Production Economics, v. 56-57, p. 199-212, 1998. http://dx.doi.org/10.1016/S0925-5273(97)00009-1

GÖL, H.; ÇATAY, B. Third-party logistics provider selection: insights from a Turkish automotive company. Supply Chain Management: An International Journal, v. 12, n. 6, p. 379-384, 2007. http://dx.doi. org/10.1108/13598540710826290

GUARNIERIA, P.; HATAKEYAMA, K. Formalização da logística de suprimentos: caso das montadoras e fornecedores 
da indústria automotiva brasileira. Produção, v. 20, n. 2, p. 186-199, 2010.

HAERY, A. et al. Application of Association Rule Mining in Supplier Selection Criteria. Proceedings of World Academy of Science, Engineering and Technology, v. 3, p. 358$362,2008$.

HANDFIELD, R. et al. Applying environmental criteria to supplier assessment: A study in the application of the Analytical Hierarchy Process. European Journal of Operational Research, v. 141, p. 70-87, 2002. http:// dx.doi.org/10.1016/S0377-2217(01)00261-2

HUMPHREYSA, P. et al. Integrating design metrics within the early supplier selection process. Journal of Purchasing \& Supply Management, v. 13, p. 42-52, 2007. http://dx.doi. org/10.1016/j.pursup.2007.03.006

JAYARAMAN, V.; SRIVASTAVA, R.; BENTON, W. C. Supplier Selection and Order Quantity Allocation: A Comprehensive Model. Journal of Supply Chain Management, v. 35, n. 2, p. 50-58, 1999. http://dx.doi.org/10.1111/j.1745493X.1999.tb00237.x

JOLAl, F. et al. Integrating fuzzy TOPSIS and multi- period goal programming for purchasing multiple products from multiple suppliers. Journal of Purchasing \& Supply Management, v. 17, p. 42-53, 2011. http://dx.doi. org/10.1016/j.pursup.2010.06.004

KAKOURIS, A. P.; POLYCHRONOPOULOS, G.; BINIORIS, S. Outsourcing decisions and the purchasing process: a systems-oriented approach. Marketing Intelligence \& Planning, v. 24, n. 7, 2006 http://dx.doi. org/10.1108/02634500610711879

KRAUSE, D. R.; PAGELL, M.; CURKOVIC, S. Toward a measure of competitive priorities for purchasing. Journal of Operations Management, v. 19, p. 497-512, 2001. http:// dx.doi.org/10.1016/S0272-6963(01)00047-X

LEE, A. H. 1. A fuzzy supplier selection model with the consideration of benefits, opportunities, costs and risks. Expert Systems with Applications, v. 36, p. 28792893, 2009. http://dx.doi.org/10.1016/j.eswa.2008.01.045

LEVARY, R. R. Ranking foreign suppliers based on supply risk. Supply Chain Management: An International Journal, v. 12, n. 6, p. 392-394, 2007. http://dx.doi. org/10.1108/13598540710826317

LIAO, C. N.; KAO, H. P. An integrated fuzzy TOPSIS and MCGP approach to supplier selection in supply chain management. Expert Systems with Applications, v. 38, p. 10803-10811, 2011. http://dx.doi.org/10.1016/j. eswa.2011.02.031

LIAOA, Z.; RITTSCHER, J. A multi-objective supplier selection model under stochastic demand conditions. International Journal of Production Economics, v. 105, p. 150159, 2007. http://dx.doi.org/10.1016/j.jpe.2006.03.001

LIU, J.; DING, F.; LALL, V. Using data envelopment analysis to compare suppliers for supplier selection and performance improvement. Supply Chain Management: An International Journal, v. 5, n. 3, p. 143-150, 2000. http://dx.doi. org/10.1108/13598540010338893

LIN, T.; CHENG, Y; HUANG, M. The impact of supplier selection factor between supply chain management and human resource development. In: INTERNATIONAL CONFERENCE ON BUSINESS AND INFORMATION, 2008, Seoul. Proceedings... ATISR, 2008. Disponível em: <academicpapers.org/ocs2/session/Papers/Poster/368.doc>. Acesso em: 22 jan. 2009.

MARTIN, C. The Agile Supply Chain: Competing in Volatile Markets. Industrial Marketing Management, v. 29, n. 1, p. $37-44,2000$.
McDERMOTT, C.; HANDFIELD, R. Concurrent development and strategic outsourcing: do the rules change in Breakthrough innovation? The Journal of High Technology Management Research, v. 11, n. 1, p. 35-57, 2000. http://dx.doi. org/10.1016/S1047-8310(00)00020-1

MEADE, L.; SARKIS, J. A conceptual model for selecting and evaluating third-party reverse logistics providers. Supply Chain Management: An International Journal, v. 7, n. 5, p. 283-295, 2002. http://dx.doi. org/10.1108/13598540210447728

MURALIDHARAN, C.; ANANTHARAMAN, N.; DESHMUKH, S. G. A Multi-Criteria Group Decisionmaking for Supplier Rating. The Joumal of Supply Chain Management, v. 38 n. 4, p. 22-33, 2002. http://dx.doi.org/10.1111/j.1745493X.2002.tb00140.x

NARASIMHAN, R.; TALLURI, S.; MENDEZ, D. Supplier Evaluation and Rationalization via Data Envelopment Analysis: An Empirical Examination. The Jounal of Supply Chain Managemenrt, v. 37, n. 3, p. 28-37, 2001. http://dx.doi. org/10.1111/j.1745-493X.2001.tb00103.x

NEUMANN, C. S. R.; RIBEIRO, J. L. D. Desenvolvimento de fornecedores: um estudo de caso utilizando a troca rápida de ferramentas. Produção, v. 14, n. 1, p. 44-53, 2004 http://dx.doi.org/10.1590/S0103-65132004000100005

NG, W. L. An efficient and simple model for multiple criteria supplier selection problem. European Journal of Operational Research, v. 186, p. 1059-1067, 2008. http:// dx.doi.org/10.1016/j.ejor.2007.01.018

OZKOK, B. A.; TIRYAKI, F. A compensatory fuzzy approach to multi-objective linear supplier selection problem with multiple-item. Expert Systems with Applications, v. 38, p. 11363-11368, 2011. http://dx.doi.org/10.1016/j. eswa.2011.03.004

PEREIRA, G. M. et al. Comparando flexibilidade no produto, custos e velocidade de desenvolvimento na indústria da moda chinesa e brasileira. Produção, v. 21, n. 1, p. 27-38, 2011. http://dx.doi.org/10.1590/S010365132011005000012

PERUCIA, A.; BALESTRIN, A.; VERSCHOORE, J. Coordenação das atividades produtivas na indústria brasileira de jogos eletrônicos: hierarquia, mercado ou aliança? Produção, v. 21, n. 1, 2011.

PETRONI, A.; BRAGLIA, M. Vendor Selection Using Principal Component Analysis. Journal of Supply Chain Management, v. 36, n. 2, p. 63-69, 2000. http://dx.doi. org/10.1111/j.1745-493X.2000.tb00078.x

PIRES, S. R. 1.; SACOMANO NETO, M. Características estruturais, relacionais e gerenciais na cadeia de suprimentos de um condomínio industrial na indústria automobilística. Produção, v. 20, n. 2, p. 172-185, 2010. http://dx.doi. org/10.1590/S0103-65132010005000032

RAMANATHAN, R. Supplier selection problem: integrating DEA with the approaches of total cost of ownership and AHP. Supply Chain Management: An International Journal, v. 12 , n. 4 , p. 258-261, 2007. http://dx.doi. org/10.1108/13598540710759772

SAEN, R. F. Suppliers selection in the presence of both cardinal and ordinal data. European Journal of Operational Research, v. 183, p. 741-747, 2007. http://dx.doi. org/10.1016/j.ejor.2006.10.022

SANAYEl, A.; MOUSAVI, S. F.; YAZDANKHAH, A. Group decision making process for supplier selection with VIKOR under fuzzy environment. Expert Systems with Applications, v. 37, p. 24-30, 2010. http://dx.doi.org/10.1016/j. eswa.2009.04.063

SARKARA, A.; MOHAPATRA, P. K. J. Evaluation of supplier capability and performance: A method for supply base reduction. Journal of Purchasing \& Supply Management, 
v. 12, p. 148-163, 2006. http://dx.doi.org/10.1016/j. pursup.2006.08.003

SARKIS, J.; TALLURI, S. A model for strategic supplier selection. Journal of Supply Chain Management, v. 38, n. 1, p. 1823, 2002. http://dx.doi.org/10.1111/j.1745-493X.2002. tb00117.x

SHEMSHADI, A. et al. Fuzzy VIKOR method for supplier selection based on entropy measure for objective weighting. Expert Systems with Application, v. 38, p. 12160-12167, 2011. http://dx.doi.org/10.1016/j.eswa.2011.03.027

SONGHORI, M. J. et al. A supplier selection and order allocation model with multiple transportation alternatives. International Journal Advanced Manufacturing Technology, v. 52, p. 365-376, 2011. http://dx.doi. org/10.1007/s00170-010-2697-0

SZAJUBOK, N. K.; ALENCAR, L. H.; ALMEIDA, A. T. Modelo de gerenciamento de materiais na construção civil utilizando avaliação multicritério. Produção, v. 16, n. 2, p. 303-318, 2006. http://dx.doi.org/10.1590/S010365132006000200010

TAMA, M. C. Y.; TUMMALA, V. M. R. An application of the AHP in vendor selection of a telecommunications system. Omega, v. 29, p. 171-182, 2001. http://dx.doi. org/10.1016/S0305-0483(00)00039-6

TALLURI, S. A buyer-seller game model for selection and negotiation of purchasing bids. European Journal of Operational Research, v. 143, p. 171-180, 2002. http:// dx.doi.org/10.1016/S0377-2217(01)00333-2

TALLURI, S.; NARASIMHAN, R. Vendor evaluation with performance variability: A max-min approach. European Journal of Operational Research, v. 146, p. 543-552, 2003. http://dx.doi.org/10.1016/S0377-2217(02)00230-8

TALLURIA, S.; NARASIMHANA, R.; NAIR, A. Vendor performance with supply risk: A chance-constrained DEA approach. International Journal of Production Economics, v. 100 , p. $212-222$, 2006. http://dx.doi.org/10.1016/j. ijpe.2004.11.012

TING, S.; CHO, D. An integrated approach for supplier selection and purchasing decisions. Supply Chain Management: An International Journal, v. 13, n. 2, p. 116-127, 2008. http:// dx.doi.org/10.1108/13598540810860958

VERMA, R.; PULLMAN, M. E. An Analysis of the Supplier Selection Process. Omega - International Journal Management Science, v. 26, n. 6, p. 739-750, 1998.
VINCKE, P. Multicriteria decision-aid. Bruxelles: Wiley, 1992.

VOKURKA, R.; CHOOBINEH, J.; VADI, L. A prototype expert system for evaluation and selection of potential suppliers. International Journal of Operation \& Production Management, v. 16, n. 12, p.106-127, 1996. http://dx.doi. org/10.1108/01443579610151788

VONDEREMBSE, M. A.; TRACEY, M. The impact of supplier selection criteria and supplier involvement on manufacturing performance. Journal of Supply Chain Management, v. 35, n. 3, p. 33-39, 1999. http://dx.doi. org/10.1111/j.1745-493X.1999.tb00060.x

WANGA, G.; HUANG, S. H.; DISMUKESA, J. P. Product-driven supply chain selection using integrated multi-criteria decision-making methodology. International Journal of Production Economics, v. 91, p. 1-15, 2004. http://dx.doi. org/10.1016/S0925-5273(03)00221-4

WANKE, P. F.; AFFONSO, C. R. Determinantes da eficiência de escala no setor brasileiro de operadores logísticos. Produção, v. 21, n. 1, p. 53-63, 2011. http://dx.doi. org/10.1590/S0103-65132010005000045

WEBER, C. A. A data envelopment analysis approach to measuring vendor performance. Supply Chain Management, v. 1, n. 1, p. 28-39, 1996. http://dx.doi. org/10.1108/13598549610155242

WEBER, C. A.; CURRENT, J.; BENTON, W. C. Vendor selection criteria and methods. European Journal of Operational Research, v. 50, p. 2-18, 1991. http://dx.doi. org/10.1016/0377-2217(91)90033-R

YEH, W. C.; CHUANG, M. C. Using multi-objective genetic algorithm for partner selection in green supply chain problems. Expert Systems with Applications, v. 38, n. 4, p. 4244-4253, 2011. Disponivel em: <http://www.sciencedirect.com/science/article/pii/ S0957417410010481>. Acesso em: 20 jul. 2011. http:// dx.doi.org/10.1016/j.eswa.2010.09.091

\section{Agradecimentos}

Este estudo foi parcialmente apoiado pela CAPES (Coordenação de Aperfeiçoamento de Pessoal de Nível Superior) e CNPQ (Conselho Nacional de Desenvolvimento Científico e Tecnológico).

\title{
Supplier selection methodologies: a literature review
}

\begin{abstract}
Driven by the increasing supplier's involvement in the overall performance of the purchaser, companies have become more demanding in their choices, which led to the incorporation of a variety of evaluation criteria in the decision making process. The importance and complexity of the hiring decision, in turn, have encouraged the search for new tools and techniques for vendor selection. The present study conducted a literature review of works related to the topic, where articles published between 1998 and 2011 were analyzed. This review has identified trends and gaps of the recent literature, offering insights to interested parties for further application and/or improvements.
\end{abstract}

Keywords

Supplier. Supplier selection. Decision process. 